\title{
Educar para libertar: Las propuestas educacionales de los Sem-Terra en Brasil en la década de 1990
}

\author{
Clécio Ferreira Mendes ${ }^{1}$ \\ Recibido: 19 de mayo de 2017 - Aprobado: 15 de septiembre de 2017
}

\begin{abstract}
Resumen
El análisis de la ideología educacional del Movimento dos Trabalhadores Rurais Sem-Terra (MST) en Brasil, en la década del noventa, y de su proyecto pedagógico en las escuelas autónomas de sus asentamientos, abre la posibilidad de comprender los fundamentos que orientan la relación entre Educación y Movimientos Sociales. La documentación histórica y educacional del MST utilizada para este estudio está localizada en el Centro de Documentación e Memoria (CEDEM) de la UNESP. Entre los documentos utilizados más importantes están: la Série Formação, Cadernos de Educação, Boletins Educacionais y las colecciones Fazendo História, Fazendo Escola y Pra Soletrar a Liberdade. El artículo presenta un breve resumen histórico de las reformas educacionales en América Latina que influenciaron directamente la educación oficial brasileña en los noventa. Es en ese contexto que se desarrolla el proyecto educacional del MST al cual el trabajo pone su atención.
\end{abstract}

Palabras clave: educación, movimientos sociales, Sem-Terra, Brasil.

\section{Education for freedom: Educational proposals by the Sem-Terra in Brazil in the 1990s}

\begin{abstract}
The analysis of the educational ideology of the Movimento dos Trabalhadores Rurais Sem-Terra (MST) in Brazil in the Nineties and its pedagogical project at autonomous schools of its settlements, gives us the possibility of understanding the foundations guiding the relationship between Education and Social Movements. The historical and educational documentation of the MST used for this
\end{abstract}

1 Brasileño. Doctor en Historia Social por la Pontificia Universidad Católica de São Paulo, Brasil, e investigador del Centro de Estudos de História da América Latina (CEHAL) de la misma universidad. Este artículo es parte de la investigación de magíster que tiene por título "Pra soletrar a liberdade: as propostas educacionais do movimento Zapatista no México e dos Sem-Terra no Brasil na década de 90", del 2005. Dicha investigación fue financiada por la beca integral del Conselho Nacional de Desenvolvimento Científico e Tecnológico (CNPq), Brasil. E-mail: historiadorclecio@gmail.com 
study is located in the UNESP Documentation and Memory Center (CEDEM). Among the most important documents used are: Série Formação, Cadernos de Educação, Boletins Educacionais and the Fazendo História, Fazendo Escola and Pra Soletrar a Liberdade collections. The article presents a brief historical summary of the educational reforms in Latin America that directly influenced Brazilian official education in the Nineties. In this context, the educational project of the MST is developed, which is the focus of this work.

Keywords: Education, social movements, Sem-terra, Brazil.

\section{Educar para libertar: As propostas educacionais dos Sem-Terra no Brasil na década de 1990}

\section{Resumo}

A análise da ideologia educacional do Movimento dos Trabalhadores Rurais Sem-Terra (MST) no Brasil, na década de noventa, e de seu projeto pedagógico nas escolas autônomas de seus assentamentos, abre a possibilidade de compreender os fundamentos que orientam a relação entre Educação e Movimentos Sociais. A documentação histórica e educacional do MST utilizada para este estudo está localizada no Centro de Documentação e Memória (CEDEM) da UNESP. Entre os documentos mais importantes utilizados estão: a Série Formação, Cadernos de Educação, Boletins Educacionais e as coleções Fazendo História, Fazendo Escola e Pra Soletrar a Liberdade. 0 artigo apresenta um breve resumo histórico das reformas educacionais na América Latina que influenciaram diretamente a educação oficial brasileira nos anos noventa. É nesse contexto que se desenvolve o projeto educacional do MST ao qual o trabalho põe sua atenção.

Palavras-chave: educação, movimentos sociais, Sem-terra, Brasil

"Somos un Movimiento de masas, de carácter sindical, popular y político. Luchamos por tierra, reforma agraria y cambios en la sociedad"2 (Almeida y Sánchez, 1998: 81).

La cita da cuenta de la autodefinición asumida por el MST, cuya organización ocurre al comenzar el año 1984 al interior del cuadro socio-político originado entre 1978 y 1980, marcado por el proceso de redemocratización de Brasil, el surgimiento del nuevo sindicalismo, de los movimientos sociales urbanos y de las Comunidades Eclesiales de Base (CEB), entre otros. Dentro de ese proceso, los campesinos brasileños reiniciaron la lucha histórica por la conquista de la tierra a través de ocupaciones territoriales organizadas por centenas de familias, las que se agruparon en campamentos y luego en

2 Traducciones de citas al español del autor. 
asentamientos que se replicaron por todo Brasil. En 1984 los diversos grupos a lo largo del país realizaron un encuentro y articularon el nacimiento del Movimento dos Trabalhadores Rurais Sem-Terra (em adelante, MST). "El Movimiento está organizado en 23 estados brasileños, involucrando 1,5 millón de personas, con 300 mil familias asentadas y 80 mil que todavía viven en campamentos" (MST: 2003).

El MST, en sus más de treinta años de existencia, tiene como sus principales objetivos: la reforma agraria, una sociedad más justa, la expropiación de las grandes áreas en manos de las multinacionales y el fin de los latifundios improductivos, con la definición de un área máxima de hectáreas para la propiedad rural y la autonomía para las áreas indígenas, manifestándose en contra de la revisión de las tierras de esos pueblos. ${ }^{3}$ La tierra es el tema central del Movimiento y su comprensión demanda una retrospectiva histórica, dado que tales luchas no son recientes aunque lo sean en su forma actual. En Brasil el tema de la tierra se instala desde tiempos inmemoriales, agudizándose con la inexistencia de una reforma agraria que, por lo menos, atendiese las exigencias del capitalismo. Los problemas de los trabajadores rurales también tienen raíces históricas, agravadas por su exclusión en las legislaciones laborales del período de Getúlio Vargas (1930-1945), y por el modo de producción monopolista que caracteriza el campo. A esto se suma, en los últimos años, el atávico desempleo que hace que innumerables familias vaguen por el país tras la búsqueda de condiciones de sobrevivencia.

EI MST nace desde la articulación de varios movimientos campesinos que surgieron o se fortalecieron en varias regiones de Brasil. Esa articulación ocurre fundamentalmente a partir del trabajo de acercamiento de esos movimientos realizado por la Pastoral de la Tierra a fines de 1970, posibilitando el surgimiento del MST en 1984, al final del régimen cívico-militar que asoló Brasil hasta 1985 y cuyo proceso de distensión rumbo a la democracia mantuvo hasta hoy enclaves dictatoriales que se manifiestan de varias formas y acciones del Estado. Este contexto también está marcado por el fortalecimiento de los movimientos sociales y sindicales que generaron nuevas fuerzas políticas en el país que influenciaron directamente los rumbos históricos de Brasil, como el surgimiento del Partido dos Trabajadores (PT) en 1980 y de la Central Única dos Trabajadores (CUT), también en el inicio de la misma década.

EI MST, junto a su avance y crecimiento, desarrolla otras demandas que se suman a las construidas históricamente y nacionalmente, como la mejora de la producción en los asentamientos, el acceso a la escuela para todas

3 La revisión es la redefinición del área que puede ser ocupada por los indígenas. En Brasil muchas de las tierras indígenas despiertan intereses económicos por la extracción de madera, minería y creación de ganado. 
las edades y eliminar el hambre, entre otras. La percepción del movimiento era que la formación política, inherente a la lucha social, necesitaba ser acompañada por una formación educacional que permitiera el desarrollo intelectual, político y técnico continuo al interior del movimiento. Conciben que es necesario desarrollarse más allá de la reforma agraria para ampliar y consolidar su lucha.

Nuestra investigación se aboca a la búsqueda de los principios filosóficos y educacionales que orientan el proyecto educacional del MST. Una propuesta que nace desde la demanda social del propio movimiento y que no encuentra en la escuela oficial, del modelo neoliberal, la identificación cultural e ideológica. Para desarrollar sus escuelas autónomas, se instalan desde la perspectiva de buscar los derechos de los campesinos, entendiendo la educación como una forma de concientización popular y un medio para la construcción de nuevas formas de relaciones sociales.

\section{Las reformas educacionales: La trama neoliberal en América Latina en los años noventa}

La intención de ese ítem es enunciar en qué consistieron las reformas educacionales gubernamentales de la década del noventa en América Latina, sus objetivos y su relación con el neoliberalismo. Brasil y otros países de la región participaron de un proceso común y adecuaron sus sistemas oficiales de educación a las nuevas determinaciones de la economía mundial en la post bipolarización del mundo.

Son diversos los autores que analizan los problemas educacionales de América Latina, la relación del Banco Mundial con las reformas educacionales y las particularidades e iniciativas regionales en educación. Algunos de esos autores han contribuido directamente, como Pablo Gentili, Francisco de Alencar y Gaudêncio Frigotto, entre otros; enfatizando el carácter impositivo de esas reformas y la contradicción entre un discurso descentralizador y de autonomía, que debería resguardar las identidades locales y/o regionales. También destacan los propósitos de las reformas, analizando sus implicaciones y las formas de su implementación.

Al hablar de los objetivos de las reformas educacionales, necesitamos identificar quiénes las reivindican o quiénes determinan cómo van a suceder. En el caso de América Latina, se observa que el Estado las determina y encamina a través de sus ministerios y secretarías de educación, conforme evaluaciones externas a las comunidades escolares y, muchas veces, al propio Estado. No obstante, el discurso corriente era que la educación propiciaría una transformación social significativa, una vez que posibilitaría a los individuos la condición para que transformasen sus vidas y solucionaran sus problemas de 
sobrevivencia e integración social. Apunta, inclusive, que la educación sería la responsable de los problemas sociales vividos en América Latina.

El análisis del desempleo (un problema social) está estrechamente relacionado a la falta de calificación de mano de obra, por tanto, a la población que no responde a las necesidades del mercado laboral. El problema del desempleo dejaría de ser estructural y se tornaría educacional, por lo que el Estado debería enfocarse a la formación de fuerza de trabajo calificada que atienda el mercado. Pero esa perspectiva no es compartida por los Sem-Terra. En los documentos producidos por el MST sobre educación hay críticas que cuestionan la calidad y el contenido político-social de los programas oficiales de la educación del Brasil, que seguía los órdenes de las reformas apuntadas hacia la América Latina. El movimiento enfatiza la crisis de la educación y la falta de atención a las necesidades de las comunidades, principalmente con relación a una educación propia del campo.

Para comprender el contexto de las críticas del MST con relación al modelo educacional brasileño en los noventas, es importante observar los cambios de la economía-política en América Latina. En ese sentido, Pablo Gentili apunta al Consenso de Washington, un núcleo de doctrinas económicas y financieras resultante de un programa de ajustes y estabilización, "como un sentido común de administraciones gubernamentales latinoamericanas" (Gentili, 2002: 14). Sus orientaciones han sido aplicadas para solucionar el problema del déficit público y de la estabilización de las economías, siendo justificadas por los gobiernos cuando los organismos financieros (como el Banco Mundial y el FMI) exigen garantías para realizar préstamos. El autor todavía apunta que las "elites políticas y económicas locales" adoptaron lo que él nombra "ortodoxia neoliberal" (Gentili, 2002: 14), alternativa promocionada por esos organismos como el único camino para salir de la crisis provocada por las deudas contraídas por los países latinoamericanos, a pesar de las fuertes críticas de diversos autores sobre la eficacia de tales medidas para la solución de los problemas apuntados.

Del mismo modo que hubo una homogeneización en el proceso de estabilización y de reformas económicas entre los gobiernos latinoamericanos, hubo también una padronización de la reforma educacional, percibida en la regularidad de la:

"expansión de un mismo núcleo de diagnósticos, propuestas y argumentos 'oficiales' acerca de la crisis educacional y de sus supuestas salidas, así como en la circulación y en el impacto (directo e indirecto) que los documentos y 'recomendaciones' del Banco Mundial y del FMI tienen en la definición de las políticas destinadas a este sector. Por lo tanto, hay un 'nuevo sentido común tecnocrático', que también penetró capilarmente en los Ministerios de Educación" (Gentili, 2002: 15). 
Eso conduce, conforme el autor, los "diagnósticos y decisiones políticas de los administradores del sistema escolar" (Gentili, 2002: 15). A partir de esa idea común, era recurrente en América Latina un discurso oficial categórico en defensa de las reformas educacionales. Esas, se decía, estaban direccionadas hacia la calidad de la enseñanza y el combate a las crisis del sector, y figuraban como propulsores de las transformaciones sociales necesarias para el avance de la región rumbo a su compatibilización con los países más industrializados.

Otras lecturas que analizan la historia de la educación cuestionan la idea de que ella sea el agente transformador de una sociedad. Es el caso de Aníbal Ponce, en "Educación y lucha de clases", que apunta esa creencia como:

"un desconocimiento absoluto de la realidad social. Ligada estrechamente a la estructura económica de las clases sociales, la educación, en cada período histórico, no puede ser otra cosa que un reflejo necesario y fatal de los intereses y aspiraciones de esas clases" (Ponce, 2003: 168).

En la lectura de los análisis de los movimientos y de la crítica bibliográfica con relación a la educación, es importante destacar que los objetivos y resultados alcanzados muestran los reales intereses sociales involucrados: la mantención del statu quo y de la producción de mano de obra barata y abundante para un mercado de trabajo limitado, además de una pretenciosa desvinculación de la crisis social latinoamericana provocada por el mecanismo de funcionamiento del sistema capitalista.

Dentro del mecanismo de funcionamiento del capitalismo, la educación ejerce un papel estratégico, a través del cual las clases dominantes gestan parte de las condiciones de su existencia a través de la formación de las mentalidades y de las conductas de los seres sociales. Es necesario comprender la educación no como el único medio por el cual se da la dominación política, económica y cultural del pueblo, sino también como la gestión de las condiciones de existencia de las clases dominantes, como fue mencionado anteriormente, comprendiendo que la educación ejerce la función de transmisor de valores y conocimientos acumulados y sistematizados por la sociedad. Por tanto, vale decir:

"la educación es el proceso mediante el cual las clases dominantes preparan en la mentalidad y en la conducta de los niños las condiciones fundamentales de su propia existencia. Pedirle al Estado que deje de interferir en la educación es lo mismo que pedirle que proceda de esa forma en relación con el Ejército, la Policía y la Justicia" (Ponce, 2003: 169). 
Se observa un distanciamiento de las propuestas de las reformas educacionales en relación a la realidad vivenciada por las escuelas. Esto complica el acceso de los propios estudiantes y el combate a las dificultades enfrentadas por los profesionales que allí actúan, interfiriendo en el funcionamiento de las clases y de todo el proceso de enseñanza. Por eso, hay críticas a los análisis y a los diagnósticos oficiales sobre el sistema educacional en el continente, que, conforme se observa en el texto de Candau, son:

"orientados por técnicos de organismos internacionales y por profesionales locales, generalmente basados en enfoques economicistas y centrados en el tema de la productividad y de la necesidad de generar reformas educativas que favorezcan la inserción de los respectivos países en la lógica de la competitividad, imprescindible en un mundo cada vez más globalizado y regido por el Libre Mercado" (Candau, 2000: 78).

Siguiendo la lógica del discurso neoliberal, no faltan escuelas, profesores ni recursos, pero sí una administración adecuada sumada a un cambio sustantivo en las prácticas pedagógicas que las tornen eficientes y puedan alcanzar resultados satisfactorios. Podemos observar estas orientaciones en el siguiente párrafo del documento de Preal de 1996:

"Actualmente, existe consenso en relación al punto de vista de que la apertura al comercio internacional es algo necesario y positivo. Varios países se han beneficiado enormemente aplicando esta política económica, aprovechando sus ventajas competitivas. (...) En la competencia entre países, existen algunos que están consiguiendo producir más con menos personas calificadas. (...) El problema es cómo obtener recursos humanos adecuados a esos nuevos desafíos, recursos capaces de adaptarse a los cambios que vendrán en el futuro. La respuesta se obtiene a través de un currículo educativo moderno, sintonizado con las necesidades del mundo del trabajo. Creo que debemos ocuparnos muy seriamente de las competencias exigidas actualmente por el mundo del trabajo, que son radicalmente distintas de las existentes en épocas pasadas; (...) se exige gente mucho más flexible, con una gran autonomía y con grandes capacidades de comunicación" (Preal, 1998: 20).

A través de la orientación de Preal, se verifica claramente el enfoque economicista dado a la educación, relacionado solamente a la productividad y a la competitividad en detrimento del énfasis en la formación de valores, actitudes y comportamiento que generan individuos capaces de elaborar propuestas transformadoras de la realidad y no solo sumar a lo que ya existe. De esa forma, los organismos internacionales se limitaron a afirmar que la reforma educativa era fundamental para el desarrollo social, político y eco- 
nómico de la América Latina, sin considerar las relaciones sociales, políticas y económicas vigentes en cada país de la región. Estas propuestas asociaban la educación al desarrollo financiero y económico, es decir, con el mercado.

Dada la importancia que asumen tales organismos en la región, es natural que uno de los debates más frecuentes sea el papel que dichos organismos han ejercido en las políticas educacionales, particularmente el Banco Mundial, por el número de préstamos que determina el contenido del proceso que orientó las reformas. La actuación de este Banco trajo consecuencias directas al mundo, no siempre positivas. Según Maria Clara Couto Soares:

"Después de 50 años de operación y préstamos de más de US\$250 billones, la evaluación de la performance del Banco Mundial es extremamente negativa. Esta financió un tipo de desarrollo económico desigual y perverso socialmente, que amplió la pobreza mundial, concentró la renta, profundizó la exclusión y destruyó el medio ambiente" (Tommasi, 1998: 17).

En Brasil esas políticas vienen ejerciendo una influencia profunda desde el período de la dictadura cívico-militar, más específicamente en la década de 1970, ya que el Banco financió el proyecto de industrialización implementado por la burguesía. En los años 1980, el Banco impuso condiciones como garantías para los préstamos que involucraban programas de ajustes de la economía, en conjunto con el FMl, interfiriendo directamente en la gobernabilidad. El resultado de tales políticas conllevó un agravamiento de los problemas sociales y la ampliación de las desigualdades, no solamente en el interior de países similares, sino también entre las naciones desarrolladas.

En conformidad con las nuevas directrices del Estado, la reforma educacional fundamentó y consolidó los objetivos de constituir relaciones de mercado y de competitividad en la formación del educando, con vistas a establecer un nuevo parámetro de ciudadano:

"un nuevo modelo de regulación que viene instaurándose en la organización y gobernación (entendida como la acción de gobernar) de la educación pública -tanto del sistema como en sus instituciones en América Latina, a pesar de ciertas especificidades en sus distintos países-, formas casi mercantiles de delegación de poderes y en las relaciones con la demanda educacional, como adoptar el principio de mercado como indicador de las realizaciones en todas las esferas sociales y también al resignificar el concepto de ciudadanía en cuanto consumo" (Vieira; Krawczyk, 2003: 118).

El resultado fue que esas políticas asumidas por los gobiernos nacionales, como en el caso de Brasil, contribuyeron al agravamiento de las cuestiones 
sociales y al empobrecimiento de la población. Es en el contexto de esas decisiones nacionales e internacionales, particularmente en el ámbito de la economía, que se insertaron las reformas educacionales. Su objetivo era producir un tipo de desarrollo que atendiera las necesidades del mercado de trabajo y de la población hasta en un nivel internacional, sin importar que el efecto de esa política fuese la exclusión de un enorme contingente poblacional.

Las propuestas no respetaron las particularidades y las necesidades regionales. Incluso, la reforma educacional propuso la descentralización, visando dar autonomía a las escuelas para que definieran sus contenidos programáticos. Sin embargo, factores diversos llevaron a la centralización: los libros didácticos, el material de apoyo, la malla curricular, el calendario académico y otras que fueron definidas por la administración central, incluso los criterios de evaluación de desempeño de los estudiantes. Esos factores acabaron por concretizar un proceso centralizado, que dejó muy poco espacio para propuestas alternativas.

En Brasil los Parámetros Curriculares Nacionales (PCN) se presentan como orientaciones supuestamente flexibles y no obligatorias a los profesores. No obstante, son muchos los autores que denuncian el carácter autoritario y uniformizado de los PCN, que no respetan la diversidad cultural y social de este país-continente. De esa forma, las propuestas educacionales de los movimientos sociales fueron fruto de un embate a la visión neocolonial de la educación, que debe atender a los intereses del mercado de trabajo y a los de una sociedad que valora la eficiencia y la productividad.

Entre los diversos movimientos sociales que han tenido más impacto político en América Latina, el MST se destaca como referencia en la resistencia al neoliberalismo que se impuso en Brasil en la década del noventa. Y confronta el modelo educacional oficial con una concepción de educación que persigue el desarrollo de la humanidad y la resistencia social.

\section{El problema educacional brasileño: la mirada del MST}

El debate educacional nunca fue tan valorado como en los últimos tiempos en Brasil. Desde la victoria de Fernando Henrique Cardoso en la elección presidencial de 1994, la propuesta de cambio o modernización asumió la pauta de las políticas educacionales. La crisis en la educación es identificada con la falta de eficiencia de las instituciones escolares, siendo necesario adecuarlas a una serie de exigencias del mundo actual. De esa forma, la palabra de orden era "reformar". Los neoliberales entienden que esta reforma debe transferir la educación de la esfera del derecho a la del mercado. Solamente de esa manera se alcanzaría la eficacia y productividad necesarias en el sector. 
Toda esa voluntad política se presentó en un enorme conjunto de medidas que incluyeron reestructuración de escuelas, legislación, municipalización y programas de capacitación de profesores, entre otras propuestas. Pero en lo que se refiere al cotidiano de la escuela real, poco se alteró, porque:

"La calidad de lo que es enseñado también tiene que ver con la repitencia y la deserción: ¿Cómo no salir de una escuela donde la realidad, con su cruz y su luz, no entra? ¿Cómo disfrutar la aventura de la lectura de libros que hablan de otro mundo, distante y extraño? Quien no lee sabe menos, y nuestras elites quieren exactamente eso: a lo más, un mínimo de adiestramiento técnico. Nada de desafíos ni cuestionamientos. Quien está desinformado es más fácilmente explotado" (Gentili; Alencar, 2001: 61).

Así, la crisis en la educación se perpetúa y se afirma como forma de exclusión social, resultado directo de la aplicación de las propuestas neoliberales. Cuando el estudiante no se reconoce en esa escuela, la propia institución logra crear mecanismos de expulsión automática.

Es en ese contexto que podemos entender las propuestas educacionales de los Sem-Terra en Brasil. Además de la propia necesidad de educar niños y jóvenes pertenecientes al movimiento y la búsqueda de calificarse políticamente, también, a partir del análisis de la realidad educacional brasileña, es que surge la cobranza del Movimiento por la atención de las demandas del pueblo campesino.

"Al comienzo los Sem-Terra creían que organizarse para luchar por la escuela era apenas una más de sus luchas por derechos sociales; derechos de los que estaban siendo excluidos por su condición de trabajadores sin tierra. Pero luego fueron percibiendo que se trataba de algo más complejo. Primero porque había (como hay hasta hoy) muchas familias trabajadoras del campo y de la ciudad que tampoco tenían acceso a este derecho. Segundo, e igualmente grave, se dieron cuenta de que solamente tendrían un lugar en la escuela si buscasen transformarla" (Caldart, 2000: 45).

El análisis de la realidad fue proporcionando al MST una mayor conciencia de la importancia y de la situación de la educación nacional. Era necesario asumir la tarea de organizar, articular y producir una propuesta pedagógica para la especificidad vivida por el pueblo del campo. Entonces, en 1994 se inició una encuesta en los asentamientos y campamentos del MST que demostró índices alarmantes en el ámbito educacional. El analfabetismo era de $29 \%$. En relación a los niños, solo 1,6\% terminaba la enseñanza fundamental, y el peor de los indicadores era que el $20 \%$ de los niños y $70 \%$ de los adultos no tenían acceso a la escuela (UNESP: 1995). Las dimensiones de 
la lucha por educación que había comenzado en 1987 con la formación del Sector de Educación, se ampliaron con el objetivo de superar esta situación de exclusión y de crear una escuela que atendiera a la población campesina. Ese es un importante análisis crítico que el Movimiento hizo de la escuela: ella se distancia del campo en sus prácticas y propuestas pedagógicas y no dialoga con el Sem-Terra.

"Fueron descubriendo, de a poco, que las escuelas tradicionales no tienen lugar para sujetos como los Sem-Terra, así como tampoco acostumbra tener lugar para otros sujetos del campo, ya sea porque su estructura formal no permite su ingreso o porque su pedagogía no respeta o desconoce su realidad, sus saberes, su forma de aprender y de enseñar" (Caldart, 2000: 45).

La crítica a la escuela oficial se dirige, antes de todo, a su formato tradicional y a su estructura rígida, que no permite el acceso. Y lo que es más grave, a la permanencia de los estudiantes en su interior. La escuela, que debería ser un local privilegiado de lucha por las conquistas por derechos, de democratización del conocimiento y surgimiento de nuevas prácticas sociales, acaba despolitizada y apartando cualquier posibilidad de cambio social. La idea de una educación del campo tiene su origen en la comprensión de que la situación efectiva de sus familias empeoró debido, entre otros factores, a la "ausencia de políticas públicas que garantizaran el derecho a la educación y la escuela para los campesinos/trabajadores del campo" (Caldart, 2004: 18).

Constatado el problema educacional, se toma la decisión sobre cuál será la acción para intervenir ese escenario y conquistar los derechos sociales. Para Pablo Gentili, "la lucha contra el monopolio del conocimiento constituye un momento central en la posibilidad de conquista de sus derechos" (Gentili, 1998: 122). De ese modo es que diseñan los primeros pasos hacia la:

"lucha del pueblo del campo por políticas que garantizaran su derecho a la educación y a una educación que sea del campo. Uno: el pueblo tiene derecho a ser educado en el lugar donde vive. Dos: el pueblo tiene derecho a una educación pensada desde su lugar y con participación, vinculada a su cultura y a sus necesidades humanas y sociales" (Caldart, 2000: 25-6).

La educación del campo pasa a tener un rol fundamental en la construcción de esa escuela que busca la formación plena del ser humano. El proyecto se materializa en la colección de libros titulada Por Uma Educação do Campo, en que los integrantes y los llamados Amigos do MST analizan la educación y elaboran propuestas para la superación de su situación actual. "Nuestra lucha es en el campo de las políticas públicas, porque esta es la 
única manera de universalizar el acceso de todo el pueblo a la educación" (Caldart, 2000: 27).

Analizando la esencia de este proyecto, percibimos que ellos amplían la comprensión del problema educacional brasileño, lo que ocasionó una redefinición de los principios educacionales del MST. En el inicio, los puntos esenciales de la propuesta en el área de la educación eran: preparación de los niños para el trabajo rural, capacitación del niño para la cooperación, construir una escuela que fuera un lugar de reflexión y calificación del trabajo productivo; en fin, propuestas que tenían una visión esencialmente política. Lentamente, el análisis de la educación pasó a ser realizado con más profundidad y la lectura de la realidad educacional brasileña, aumentando la exigencia de que la escuela atendiese las demandas del campo.

La constatación de que la situación de la educación brasileña es perversa y de que las instituciones escolares, en su estructura rígida y tradicional, niegan a los estudiantes el derecho de permanecer en su interior, se manifiesta en el hecho de que muchas veces no trabajan desde la realidad social y cultural de los estudiantes. En el caso de los niños campesinos hay, por ejemplo, al menos dos problemas: la calendarización de las clases no es compatible con la rutina del campo, como en los periodos de cosecha que cambian el ritmo de las familias; o que las municipalidades y el Estado insertan estos niños en escuelas urbanas distantes de sus casas, aplicando como única política para la educación del campo la compra de microbuses para transportarlos.

Ese cuadro trajo la necesidad de una actuación contundente del MST. La lucha por la escuela se volvió una importante bandera del Movimiento: ocupar las escuelas y transformarlas, tal como se lucha por la tierra.

\section{Para deletrear la libertad: la función social de la educación y la construcción de los proyectos educacionales}

A lo largo de su trayectoria el MST evaluó que era necesario crear un espacio de convivencia y de relaciones culturales y educacionales, con el objetivo de ampliar y fortalecer su lucha. Estos espacios serían las escuelas autónomas del Movimiento, alojadas en los asentamientos o a través de escuelas itinerantes, en el caso de los campamentos.

EI MST creó un sector específico para trabajar sobre el derecho a la educación, que involucró los veintitrés estados donde hay presencia del Movimiento. La organización de ese sector fue hecha por el Colectivo Nacional de Educación, con la participación de representantes de los estados. Partiendo 
desde las demandas, se discute cuál será la línea de acción y cuáles serán sus directrices. Los encuentros para esas discusiones ocurren por lo menos tres veces durante el año. Es importante destacar que el trabajo de los educadores es voluntario y las condiciones físicas de las escuelas son precarias, muchas veces funcionando en pequeños espacios improvisados, como barracas de lona (carpas de plástico negro).

Los números alcanzados en la historia del sector de educación dan la dimensión de la amplitud del proyecto. Actualmente existen cerca de 1800 escuelas de Enseñanza Fundamental (básicas) con 200 mil niños y adolescentes estudiando y aproximadamente 3900 educadores. Además de 250 educadores en las Cirandas Infantis, destinadas a la educación de niños hasta los seis años. También se realiza el trabajo de alfabetización de adultos por aproximadamente tres mil educadores que atingen a 50 mil alfabetizandos. Sumada la enseñanza media, el Movimiento logró construir una estructura que envuelve en total más de 380 mil estudiantes y cerca de 1.500 profesores. Además, el sector, con el apoyo de algunas Universidades federales, promueve anualmente la capacitación en nivel técnico de cerca de tres mil personas, en las carreras de magisterio y administración de cooperativas.

Según el relato del propio Movimiento, el proyecto no abarca la totalidad de los niños y adolescentes que están fuera de la escuela, principalmente por el no reconocimiento legal de su escuela. En la evaluación del MST, eso ocurre por el hecho de no aceptar una propuesta curricular oficial que no esté direccionada hacia una educación que dé cuenta de las necesidades de los hijos de los trabajadores rurales. Considerando tal premisa es que surge en Brasil el proyecto Por Una Educación do Campo, presentado por el Movimiento como una posibilidad de implementar una educación para la realidad del pueblo campesino. Es de ese proyecto que nació la colección Educação do Campo, constituida por varios volúmenes, resultado de las conferencias y encuentros organizados por el MST con la participación de muchos autores e intelectuales invitados que contribuyeron para ese debate.

Para el MST la educación es uno de los derechos sociales básicos y un principio de actividad política. Todas las actividades propuestas, desde las movilizaciones, marchas, caminatas, asambleas, etc., son pauteadas por el proceso de aprendizaje. Así, enseñar es un proceso que involucra a los "niños, las mujeres, la juventud, los adultos mayores, construyendo nuevas relaciones y conciencia" (MST: 2003), y todas las actividades se orientan hacia la comprensión de la realidad vivenciada por los campesinos.

Los Sem-Terra conciben la educación como un medio importante para la lucha y para la realización de sus objetivos como movimiento social. Además de la conquista del reconocimiento de su existencia y de su cultura, la garantía 
de acceso a la tierra y la transformación del hombre y de la mujer, será la creación de una visión de mundo y de valores humanos. Como visualizamos en los Principios da educação no MST:

"La escuela también es un lugar de vivir y reflexionar sobre los valores del nuevo hombre y la nueva mujer. La sociedad que tenemos infelizmente degradó nuestra humanidad y nuestras relaciones interpersonales, creando vicios como individualismo, autoritarismo, machismo y falta de solidaridad. Se necesita reeducar nuestra humanidad a través de estas nuevas generaciones formadas en la lucha. La escuela, por las experiencias de relacionamiento colectivo que proporciona a los niños y a los jóvenes, puede ayudar a desarrollar los valores de compañerismo, igualdad, de la fraternidad y el propio valor de la búsqueda colectiva y solidaria de la felicidad, a través de la lucha perseverante por la justicia y por la paz en nuestro país y en el mundo entero" (Caderno de Educación, № 8: 1996).

Una vez más los Sem-Terra se destacan como una nueva tendencia de los movimientos sociales latinoamericanos, comprendiendo la escuela y la educación como esfera de sus objetivos. La aplicación de eso en la realidad vivida por el Movimiento se da en sus propios espacios, en los asentamientos y campamentos, donde construye la búsqueda por la libertad de existir con su cultura y tradiciones.

Del análisis documental realizado, inevitablemente emergió un concepto que se destaca por la importancia e intensidad con que aparece: la libertad, presentándose como principio fundamental para el debate educacional del Movimiento. El concepto de libertad aplicado posibilita el espacio para la educación diferenciada y la construcción de una consciencia alternativa que repiense la propia sociedad en que están insertados.

En la construcción de sus propuestas educacionales, la libertad es entendida como valor educacional que representa la autenticidad, reconocimiento de la cultura, autonomía e independencia. Como evalúa el Movimiento, los sistemas educacionales oficiales no atienden a esa forma de libertad, por lo tanto, su búsqueda será una de las centralidades educacionales.

La reflexión sobre la libertad como valor educacional para el MST se fundamenta más allá de las acciones de lucha por la tierra y se consolida con el acercamiento a lecturas y sustentos teóricos, como los surgidos del pensamiento de Paulo Freire: "nos inspiramos en la obra de los grandes maestros pedagogos, que veían en la educación un camino de la verdadera liberación de la persona humana, en especial de Paulo Freire" (Caderno de Educación, Nº 8, 1996). 
Eso junto a las observaciones de João Pedro Stedile, uno de los coordinadores nacionales, mencionadas en reportaje de revista, las cuales decían que:

"durante mucho tiempo se pensó que la reforma agraria era apenas por un pedazo de tierra, pero en una sociedad moderna de nada sirve la tierra si los hijos de los trabajadores rurales no tienen acceso a la escuela y al conocimiento" (Revista Sem-Terra, 1997: 27).

Las afirmaciones de los Sem-Terra sobre la educación son públicas y accesibles, así como los principios pedagógicos y filosóficos que delinean su proyecto. La observación de las premisas puede orientar la comprensión de sus ideas educativas y permiten visualizar algunos de sus objetivos, relacionados a la lucha del Movimiento.

"Los principios filosóficos dicen respecto a nuestra visión de mundo, nuestras concepciones más generales en relación a la persona humana, a la sociedad y a lo que entendemos que sea educación. Son el fundamento de los objetivos estratégicos del trabajo educativo" (Caderno de Educación, № 8: 1996).

En la comparación con la educación oficial, se percibe que el avance educacional del Movimiento también se pautea en la idea de que el desarrollo de ese derecho no pertenece exclusivamente al Estado y, por lo tanto, no debe ser discutida y controlada apartada de las comunidades y de la sociedad en general. Hay evaluaciones de expertos sobre esa participación de la comunidad, como Miguel González Arroyo, quién acompaña el proyecto educacional de los Sem-Terra, definiéndola como avanzada en relación a la oficial-que, según afirma este mismo autor, está abandonada. "Si la educación pública campesina está abandonada, la desarrolloda por los movimientos sociales hoy es una de las fronteras más avanzadas del movimiento pedagógico brasileiro" (Arroyo, s/d: 15).

Asimismo, la idea de que la educación debe ser independiente está presente en el Movimiento. Ella es consolidada por la concepción de que la educación no está solamente vinculada al espacio escolar, sino también, y con gran fuerza, al espacio de lucha. Se educa, igualmente, en el proceso de lucha del Movimiento, involucrando a todas y todos, de todas las edades y en prácticamente todos los momentos importantes. Se constituye un cotidiano educacional más allá de las formalidades escolares, tornando la educación como un motivo más de la existencia del Movimiento.

"Una de las lecciones que podemos recoger de nuestra historia hasta aquí es que luchar solamente por la tierra no basta. La lucha por la Reforma Agraria es mucho más amplia, e implica la conquista de todos los derechos sociales que componen lo que se podría llamar 
Ciudadanía plena. Y la Educación es uno de estos derechos, por el cual también es necesaria la organización y la lucha en nuestro país. Para nosotros la Educación sucede en un proceso, desde la participación de los niños, las mujeres, de la juventud, los adultos mayores, construyendo nuevas relaciones y conciencias, hasta la participación en las marchas, asambleas, cursos, caminatas, trabajos voluntarios, gestos de solidaridad, ocupaciones, movilizaciones, reunirse para aprender y enseñar el alfabeto, y más que eso, el acto de leer y escribir la realidad y la vida" (Caderno de Educación n. 8: 1996).

Histórica e intelectualmente los Sem-Terra relacionan la educación con sus luchas y con los principales conflictos vividos por la humanidad, ampliando la idea de identificar la educación a la lucha de clases, localizando opresores y oprimidos. Apuntan la educación como parte de una sistematización política controlada de la sociedad, de la producción de la desigualdad social y de opresión. Cuando identificamos teóricamente los Sem-Terra, encontramos algunos referentes a través de los cuales buscan pensar la educación y muchas de las afirmaciones discutidas hasta ese momento. Algunos de los autores que componen esos referentes son Florestan Fernandes, Che Guevara, José Martí, Karl Marx y Aníbal Ponce, entre otros. Uno de los autores citado constantemente por el movimiento es Paulo Freire, principalmente con relación a obras como Pedagogía del Oprimido.

"De acuerdo con los ideales socialistas y colectivos, basados en el principio de solidaridad, el proyecto educacional del MST tiene como base teórica Paulo Freire, Florestan Fernandes, Che Guevara, al cubano José Martí, al ruso A. Makarenko y clásicos como Marx, Engels, Mao Tse-Tung y Gramsci" (Revista Sem-Terra, 1997: 27).

El Movimiento tiene parámetros sobre la educación que involucra también la discusión sobre cuál es su función social. Percibimos que esos parámetros representan la visión del Movimiento sobre el papel de los campamentos y asentamientos, así como lo que la educación debe generar en esos espacios, o sea, su finalidad o su filosofía de acción. En esa dirección, buscan rescatar el sentido más amplio de la educación, colocada aquí no solamente en el espacio en que se refleja el conocimiento sistematizado, sino como una actividad cotidiana, reconocida como integrante de las relaciones sociales comunes a todos $y$, por lo tanto, de responsabilidad colectiva.

Los Sem-Terra, venidos de innumerables regiones del país, tienen en común la cuestión rural, la lucha por el espacio de construcción de relaciones societarias desfiguradas por la más radical exclusión. En este sentido, el precepto educacional fundante de su filosofía es también el tema de la tierra, que para el MST se instala como el elemento común a la diversidad de conductas, valores, orígenes, culturas y otras: 
"1) La escuela necesita preparar a los niños y a los jóvenes para el medio rural. Desarrollar el amor por el trabajo de la tierra y también traer conocimientos que ayuden concretamente al asentamiento a enfrentar sus desafíos en los campos de la producción, de la educación, de la salud, de la habitación, etc. 2) La enseñanza debe comenzar en la práctica y llevar el conocimiento científico a la realidad. El punto inicial para desarrollar los contenidos de las diferentes materias de enseñanza deben ser las propias experiencias de trabajo organizado de los niños y jóvenes en el asentamiento" (Caderno de Educación, № 8, 1996).

La preocupación con la comunidad en su relación con la educación y con el conocimiento se vuelve central para discutir de qué manera se aplicará y cuál es la comprensión del concepto "educación" que debe ser aprendida, puesto que dentro de los principales objetivos está acercar la diversidad cultural a la par de fortalecer la independencia de los campamentos y asentamientos. Encontramos la función de polo de aglutinación de la comunidad en el espacio escuelar: "El alumno necesita tener voz y voto en la escuela, trayendo su saber y sus lecciones de lucha para integrar el currículo" (Caderno de Educación, № 8, 1996).

Esa dimensión dada al espacio escolar se traduce en la discusión y rediscusión cultural, lo que posibilita encontrar estrategias para la concretización de sus intencionalidades y de repensar sus acciones, en un permanente proceso de evaluación. Además, constituye una función educacional asumida colectivamente, de transformación de las comunidades, encarando nuevas organizaciones desde sus propias definiciones.

"Un gran desafío para la escuela es ayudar al desarrollo cultural del conjunto del asentamiento. La escuela no necesita restringir su actuación a la sala de clases. A través de los propios alumnos, la escuela puede participar y hasta organizar campañas de alfabetización de adultos, elaboración de periódicos de la comunidad, fiestas culturales, haciendo reflexiones sobre estas actividades para que sean una experiencia más que se integre al conjunto de las clases" (Caderno de Educación, № 8, 1996).

Se observa que este sentido comunitario y colectivo con que se denota a la escuela es más natural, o sea, para los Sem-Terra las acciones que concretizan esta intención necesitan ser denominadas, explicadas, indicadas: "hasta organizar campañas, elaborar periódicos, fiestas, etc.". Es un sentido de colectividad que se revela constructor de una identidad que valora los intereses comunes a esas personas y que se sobrepone a la diversidad de vivencias que traen de sus condiciones anteriores. La importancia de la educación para el MST se manifiesta en la prioridad que da a la escolarización de los integrantes del Movimiento, conforme destacan: 
“'La importancia de la educación para el MST se refleja en la preocupación por montar una escuela apenas se haga la ocupación de tierras. La primera cosa es definir el local donde funcionará la escuela', dice Mara de Jesús dos Santos, profesora de Ceará y miembro de la Sectorial de Educación" (Revista Sem-Terra, 1997: 27).

Incluso con la precariedad que es vivenciada por las personas que todavía no están asentadas en campamentos, su prioridad está en la organización del espacio que les posibilitará la continuidad del trabajo pedagógico, demostrando su conciencia de la necesidad de garantizar esa escolaridad y mantener el proceso de formación de sus integrantes: "los alumnos -niños y adultos- que frecuentan las escuelas rurales en los asentamientos y campamentos tienen, además del contenido normal de materias, discusiones diarias de 45 minutos sobre los acontecimientos de su cotidiano" (Revista Sem-Terra, 1997: 27).

Constituida la base de la objetivación educacional que atienda a las necesidades y cuestionamientos del pueblo, encontramos algunas de las definiciones que permiten la exteriorización de esa discusión. Incluso envolviendo el acercamiento de su propia realidad, cultura e historia, además de la concientización de su lucha. Ese trabajo debe estar contemplado en el proceso educacional, involucrando a los estudiantes de todos los niveles educacionales.

“En la organización del Movimiento, toda la sociedad sem-Terra, sea en un asentamiento o campamento, participa de la gestación de las escuelas locales. Todos deben decidir y respetar las decisiones tomadas en conjunto, ejecutar lo que fuese determinado y evaluar los resultados efectivos de cada acción colectiva. Lo mismo sucede con los alumnos dentro de las escuelas, que pueden observar sus problemas e intentar resolverlos dentro de la propia institución de enseñanza" (Revista Sem-Terra, 1997: 29).

El criterio que orienta la acción es la mayoría consensual, obtenida en reuniones. Desde el punto de vista de la función educacional de la escuela se trata de una reafirmación. Esa transformación comportamental de las comunidades una vez más cobra importancia para la constitución del propio Movimiento y se revela como un concepto de educación no encontrado, al menos frecuentemente, en la academia y en las políticas oficiales, como afirman los análisis de autoras como Renata Coltro, en reportajes de la revista Sembrando la Educación en el MST. La transformación no se pauta solamente en cambios, también en el rescate cultural, de identidad y de dignidad, por lo tanto, en el cambio del contexto que oprime. 
Se le define un papel fundamental y de largo alcance a la educación, con función de cambio, dentro de un proceso de lucha y de decisiones del Movimiento:

"Luego de las primeras ocupaciones de tierra realizadas por los trabajadores al final de la década del 70 e inicio de la década del 80, surgió la preocupación con la educación. Con la conquista de las primeras áreas de asentamiento la preocupación de garantizar el acceso a la escuela continuó. En 1983, comenzó a funcionar la primera escuela en área de asentamiento, en Ronda Alta - RS" (MST: 2001).

Junto con la lucha por el acceso a la escuela, se forjó un proyecto pedagógico que tenía como base la realidad vivenciada:

"De esta manera, el sector fue consolidado a través del eje escolar. No obstante, el proceso de construcción nos mostró que la cuestión educacional es más amplia y está presente en el cotidiano de los campamentos y asentamientos: es lo que llamamos procesos educativos, que comienzan desde el momento que las personas entran a la lucha por la tierra" (MST, 2001).

La consolidación del proceso educativo posibilitó la creación del Sector de Educación con actividades en varios frentes: alfabetización de niños, jóvenes y adultos, trabajo con la enseñanza fundamental y media, además de propiciar la formación de educadores para que actuaran directamente sobre la realidad.

"El Sector de Educación tiene una demanda muy amplia y actúa en los siguientes frentes: escuelas de primer grado de los asentamientos; escuelas (legales o no) de los campamentos; alfabetización y posalfabetización de jóvenes y adultos en los campamentos y asentamientos; educación infantil ( 0 a 6 años) en las familias; en las Cirandas Infantiles y en las pre-escuelas; escolarización de la militancia en cursos suplementarios o en cursos alternativos de $1^{\circ}, 2^{\circ}$ y $3^{\circ}$ grado; cursos de formación de profesores, de monitores, de educadores infantiles y de otros formadores" (MST: 2001).

El avance de ese sector puede ser reconocido en el proyecto que involucró el MST al inicio de los años 2000 y que se consolidó: Escuela Nacional Florestan Fernandes, que objetiva instituir un "modelo de escuela pública, gratuita y de calidad (conforme era definido por el gran sociólogo Florestan Fernandes, que da nombre a nuestra escuela)" (MST: 2001). La construcción de la escuela fue hecha a través del trabajo voluntario de los jóvenes acampados y asentados. Los jóvenes involucrados en ese trabajo adquirieron conocimientos del proceso de construcción y durante la noche estudiaban la coyuntura nacional, cooperativismo, historia, organización del MST y otros temas que surgieron. 
El proyecto político-pedagógico del Movimiento es reconocido internacionalmente, sea por la conquista de premios o por el reconocimiento de la lucha por segmentos sociales más claros. Su actuación es reconocidamente importante para evitar la total exclusión social de niños, jóvenes y adolescentes del acceso a la educación. Pero el proyecto queda restringido a los integrantes del Movimiento, no siendo divulgado por la prensa ni contemplado en la elaboración de las directrices gubernamentales. En Brasil se encuentran estudios académicos que presentan la preocupación por rescatar ese trabajo, incluso con la inserción de los textos producidos por el Movimiento.

La construcción de la estructura educacional del MST fue inicialmente direccionada a los niños y jóvenes del movimiento no asistidos por la educación oficial, que no tenía como política el desarrollo de la educación del campo. Ese proceso siguió como crítica a la estructura deficitaria de atención al estudiante del campo. También nació de una concepción ideológica del movimiento sobre la sociedad contemporánea, por lo tanto como crítica a la estructura y al sentido social de la educación oficial y sus reformas que se siguieron. En ese sentido, podemos decir que el MST piensa la educación desde una demanda objetiva y crítica de la educación oficial basada en las reformas neoliberales que se presentaron en los noventa, pero entender sus demandas objetivas como utilitaristas, por ejemplo, con relación a la necesidad de escuelas para su continuidad, es analizar el proyecto del movimiento desde una visón liberal, con fuerte tendencia hacia la derecha política que tiene la intención de quitar el contenido social y político del movimiento. Podríamos decir que se trata de una depreciación de su proyecto educacional.

EL MST constituye una de las formas alternativas de lucha en América Latina contemporánea porque apunta hacia la izquierda desde un nuevo camino para la emancipación política y humana. La participación de base popular en la construcción del Movimiento también está presente en las decisiones políticas que son llevadas a cabo por ellos. Eso se debe, principalmente, al acercamiento de la lucha política con la realidad vivida por las personas que lo componen. La legitimidad popular consolida una diferenciación de los SemTerra con relación a la mayoría de los movimientos y partidos de la izquierda tradicional, que creían que el apoyo de las masas populares les ocurriría de forma natural. El Movimiento exterioriza, a través de la educación, sus valores e ideas transformadoras, buscando recuperar la ideología que expresan por medio de sus materiales. Con eso fue posible ver el posicionamiento del Movimiento y sus luchas en el intento de "deletrear la libertad" ("soletrar a liberdade"). 


\section{Referencias bibliográficas}

\section{Fuentes primarias}

a) Impresos editados por el MST

Movimento dos Trabajadores Rurais Sem-Terra (1996). Alfabetização de jovens e adultos: didática da linguagem/MST. São Paulo: Secretaria Nacional do MST.

Movimento dos Trabajadores Rurais Sem-Terra (1997). Princípios da educação no MST. São Paulo: Secretaria Nacional do MST.

Movimento dos Trabajadores Rurais Sem-Terra (1997). Setor Educação. Estórias da Rosa. São Paulo: Secretaria Nacional do MST.

Movimento dos Trabajadores Rurais Sem-Terra (1998). Nossa turma na luta pela Terra. São Paulo: Secretaria Nacional do MST.

Movimento dos Trabajadores Rurais Sem-Terra (1998). Alfabetização. São Paulo: Secretaria Nacional do MST.

Movimento dos Trabajadores Rurais Sem-Terra (1998). Escola itinerante em acampamentos do MST. Porto Alegre: MST.

\section{Fuentes Secundarias}

\section{a) Artículos}

Alencar, C. (2001). "Educação no Brasil: um breve olhar sobre o nosso lugar", en Gentili, P. y C. Alencar. Educar na esperança em tempos de desencanto. Rio de Janeiro: Vozes.

Almeida, L. F. y Sánchez, F. R. (1998). "Um grão menos amargo das ironias da história: o MST e as luchas sociais contra o neoliberalismo", en Revista Luchas Sociais. Programa de Estudos Pós-Graduados em Ciências Sociais da PUC-SP/ Neils, $N^{\circ} 5$.

Caderno No 8 (1998). Princípios da educação no MST.

Candau, M. V. (2000). "Reformas educacionais hoje na América Latina"em M. V. Candau (Org.) Currículo: políticas e práticas. São Paulo: Papirus.

Gentili, P. (1997). "Ocupar a Terra, ocupar as escolas: dez questões e uma história sobre educação e os movimentos sociais na virada do século", en A falsificação do consenso: simulacro e imposição na reforma educacional do neoliberalismo. Rio de Janeiro: Vozes. 


\section{b) Libros}

Arroyo, M. (s/f). A escola e os movimentos sociais: relativizando a escola. UFMG, doc. mimeo.

Caldart, R. S. (2000). Pedagogia do Movimento Sem-Terra. Petrópolis: Vozes.

Gentili, P. y G. Frigotto (2002). A cidadania negada: políticas de exclusão na educação e no trabalho. São Paulo: Cortez.

Ponce, A. (2003). Educação e luta de classes. São Paulo: Cortez Editora.

Tommasi, L., M. Warde y S. Haddad (Orgs.) (1998). O Banco Mundial e as políticas educacionais. São Paulo: Cortez.

Universidade Estadual Paulista (1995). MST. Pesquisa nacional por amostra da situação educacional em assentamentos e acampamentos de reforma agrária: relatório final. São Paulo: UNESP-MST.

Vieira, V. L., N. R. Krawczyk y L. E. Wanderley (2003). América Latina: estado e reformas numa perspectiva comparada. São Paulo: Cortez. 